\title{
A numerical method for phase-change problems
}

\author{
CHARN-JUNG KIM and MASSOUD KAVIANY \\ Department of Mechanical Engineering and Applied Mechanics. The University of Michigan, \\ Ann Arbor, MI 48109, U.S.A.
}

(Received 6 September 1989 and in final form 1 February 1990)

\begin{abstract}
A highly accurate and efficient finite-difference method for phase-change problems with multiple moving boundaries of irregular shape is developed by employing a coordinate transformation that immobilizes moving boundaries and preserves the conservative forms of the original governing equations. The numerical method is first presented for one-dimensional phase-change problems (involving large density variation between phases, heat generation. and multiple moving boundaries) and then extended to solve two-dimensional problems (without change of densities between phases). Numerical solutions are obtained non-iteratively using an explicit treatment of the interfacial mass and energy balances and an implicit treatment of the temperature field equations. The accuracy and flexibility of the present numerical method are verified by solving some phase-change problems and comparing the results with existing analytical. semi-analytical and numerical solutions. Results indicate that one- and two-dimensional phase-change problems can be handled easily with excellent accuracies.
\end{abstract}

\section{INTRODUCTION}

THE SOLUTION of moving boundary problems with phase changes has been of special interest due to the inherent difficulties associated with the nonlinearity of the interface conditions and the unknown locations of the moving boundaries. Exact closed-form solutions of phase-change problems are available only for a limited number of cases [I]. A variety of approximate analytic solution techniques have been developed, including the heat balance integral [2], variational [3], embedding [4], and perturbation techniques [5, 6]. In addition, efforts to solve phase-change problems numerically have produced such diverse solution methods as the enthalpy $[7,8]$, apparent heat capacity [9], isotherm migration [10], and coordinate transformation methods [11-16]; these methods have been introduced by researchers mainly to overcome the difficulties in handling moving boundaries.

Of the numerical methods, coordinate transformation techniques have been widely used because of the advantage of working with fixed domains (the moving boundaries are immobilized in the transformed coordinates) and a good review in this approach is provided in the work of Crank [17]. However, the simplification obtained by employing coordinate transformations introduces greater complexities into the transformed governing equations. Such complexities seem to become substantial with an increase in the number of moving boundaries especially for multi-dimensional problems, since the

+ For the purpose of generality, nondimensionalization which is useful in treating a particular problem is not considered in the formulation and in the presentation of the solution method. transformed equations were derived separately for each phase.

In this paper, troublesome complexities in the transformed equations are effectively eliminated through the careful use of a coordinate transformation (the well-known Landau transformation). The present transformed equations also preserve the conservative forms, which enable the mass and heat fluxes across the control volume faces to be consistent: thus the conservation principle is satisfied exactly in each phase. Furthermore, multiple moving boundaries can be treated easily since the transformation is performed only for a representative phase.

The transformed conservation equations are solved numerically with an implicit finite-difference method described in ref. [18]. Iterations characterizing a fullyimplicit method are avoided by the adoption of an efficient algorithm suggested by Sparrow and Chuck [11] which is extended here to account for density variation between phases for the case of one-dimensional (1-D) geometry. By noting that additional convective terms are created as a result of coordinate transformations [12], the performance of various numerical schemes-central difference, upwind, hybrid, and power law schemes (the details of these schemes are explained in ref. [18])-on the accuracy and efficiency are investigated in this study.

The performance characteristics of the present numerical solution method, which can be applied to a broad class of phase-change problems due to its remarkable flexibility, are demonstrated by solving specific problems that involve volumetric effects, heat generation, and multiple moving boundaries.

\section{FORMULATION IN 1-D GEOMETRY}

The present numerical formulationt is presented in this section for 1-D geometry. Extension to multi- 


\section{NOMENCLATURE}

a coefficient in finite-difference equation

$A$ dimensionless heat generation

$b$ source term

Bi Biot number

$c_{i} \quad$ specific heat

C constant

$D \quad$ diffusion conductance

$f_{i} \quad$ variable. equation (1)

$F_{i} \quad$ mass flow rate, equation (10)

$\hat{F}_{i} \quad$ mass flow rate across the interface $\hat{X}_{i}$

$h_{i} \quad$ specific enthalpy

$h_{\text {ig }} \quad$ latent heat of gas/liquid phase change

$h_{\mathrm{sf}} \quad$ latent heat of liquid/solid phase change

$h_{x} \quad$ convective heat transfer coefficient

$h^{*} \quad$ reference enthalpy

$H$ height of a cylinder

$J_{i} \quad$ energy flow rate, equation (10)

$k_{i}$ thermal conductivity

$l$ reference length

$L_{i} \quad$ absolute value of $L_{i}$ denotes a latent heat

$M_{i} \quad$ number of control volume faces within each phase

$n$ geometry index

$N$ total number of phases

$p_{i} \quad$ exponent, equation (15)

$P \quad$ Peclet number

$S_{i} \quad$ heat generation per unit volume

Ste Stefan number

$t$ time

$T_{i} \quad$ temperature distribution

$\hat{T}_{i} \quad$ temperature at the boundary $\hat{X}_{i}$

$T_{x} \quad$ temperature at infinity

$\Delta t \quad$ time increment

$u_{i} \quad$ velocity

$V_{i} \quad$ variable, equation (11)
$\hat{V}_{i} \quad \hat{X}_{i}^{n+1 /(n+1)}$

$x, y, z$ spatial coordinates

$X_{i}, Z_{i}$ variables

$\hat{X}_{i}, \hat{Z}_{i}$ positions of boundaries.

Greek symbols

$x_{i} \quad$ thermal diffusivity

$\beta_{i} \quad$ variable

$\delta_{i} \quad$ phase thickness

$\eta_{i} \quad$ transformed coordinate

$\eta_{i, j} \quad$ positions of control volume faces in cach phase, equation (15)

$\theta \quad$ angular coordinate

i. similarity constant

$\xi \quad$ transformed coordinate

$\rho$ density ratio

$\rho_{i} \quad$ density

$\tau$ dimensionless time

$\tau_{\mathrm{c}} \quad$ dimensionless freezing time

$\tau_{1 \mathrm{c}}, \tau_{2 \mathrm{c}}, \tau_{3 \mathrm{c}}$ dimensionless freezing times from ref. [22]

$\phi \quad$ superheating parameter.

Superscripts

0 quantities at time $t_{0}$

$1 / 2$ quantities at time $t_{0}+\frac{1}{2} \Delta t$ quantities at boundaries.

\section{Subscripts}

$e, w$ east and west control volume faces

E, $W$ east and west grid points

$i$ phase index

$j \quad$ node index in each phase

$m, m+1$ phase index

$P \quad$ grid points of interest. dimensional geometry is straightforward and will be presented later. It is assumed here that all phases are separated by sharp boundaries (or interfaces). Thermophysical properties are allowed to vary within each phase as well as between different phases. In particular, densities are assumed to be constant within each phase, but they may differ between phases. Therefore, I-D convective motions due to the volumetric effects can be considered.

One of the most general situations in 1-D phasechange problems is illustrated in Fig. 1. Although the

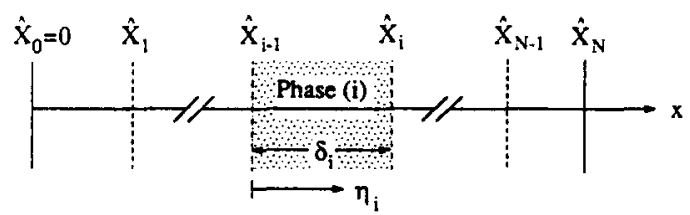

Fig. 1. Schematic of configuration considered-a system composed of $N$ distinguishable phases. configuration is shown for a planar geometry, the argument that follows is equally applicable to a cylindrical or spherical geometry.

Suppose that $N$ phases are placed along the coordinate, as shown in Fig. 1. Each boundary is either fixed or moving and can be an interface undergoing phase change or a boundary such as the edge of a thermal boundary layer and a fixed solid wall, etc.

The heat transfer process in each phase is governed by the unsteady, 1-D heat equation along with the mass continuity equation

$$
\begin{gathered}
\frac{\hat{\partial}}{\partial t}\left(x^{n} \rho_{i}\right)+\frac{\hat{c}}{\hat{\partial} x}\left(\rho_{i} f_{i}\right)=0 . \quad f_{i}=x^{n} u_{i} \\
\frac{\hat{c}}{\partial t}\left(x^{n} \rho_{i} h_{i}\right)+\frac{\hat{c}}{\hat{c} x}\left(\rho_{i} f_{i} h_{i}\right)=\frac{\hat{c}}{\hat{c} x}\left(x^{n} k_{i} \frac{\hat{c} T_{i}}{\hat{c} x}\right)+x^{n} S_{i}
\end{gathered}
$$

where $n=0,1$, and 2 for a planar. cylindrical. and 
spherical geometry, respectively, and an index $i$ that ranges from $I$ to $N$ is assigned to each phase so multiple phases can be handled with ease. The specific enthalpy $h_{i}$ is assumed to depend only on temperature. and $S_{i}$ is the heat generation per unit volume within phase $i$. The quantity $f_{i}(t)$ associated with the velocity field $u_{i}$ is included to account for any 1-D motions caused by volume changes between phases.

Now consider one of the interfaces at which a phase transition is occurring (at its phase temperature $\hat{T}_{m}$ ), and the phases adjacent to that interface. Let $\hat{X}_{m}$ be the position of the interface. The interface conditions that serve as boundary conditions of the adjacent phases are expressed as [19]

$$
\begin{gathered}
T_{m}=T_{m+1}=\hat{T}_{m} \\
\rho_{m}\left(u_{m}-\frac{\mathrm{d} \hat{X}_{m}}{\mathrm{~d} t}\right)=\rho_{m+1}\left(u_{m+1}-\frac{\mathrm{d} \hat{X}_{m}}{\mathrm{~d} t}\right) \\
\rho_{m}\left(u_{m}-\frac{\mathrm{d} \hat{X}_{m}}{\mathrm{~d} t}\right) h_{m}-k_{m} \frac{\partial T_{m}}{\hat{c} x} \\
=\rho_{m+1}\left(u_{m+1}-\frac{\mathrm{d} \hat{X}_{m}}{\mathrm{~d} t}\right) h_{m+1}-k_{m+1} \frac{\partial T_{m+1}}{\hat{c} x}
\end{gathered}
$$

where all quantities are evaluated at the interface $\hat{X}_{m}$. Equation (3) indicates the interface temperature is the temperature of phase equilibrium (for the prevailing pressure). Both the sides of equation (4) represent the mass flow rates per unit area across the interface and are equal to each other according to the conservation of mass. Equation (5) states the balance of thermal energy delivered to the interface. One of the conduction terms in equation (5) may be replaced by the convective heat fluxes or by the radiative heat fluxes, etc., depending on the problem of interest (treatment of such cases is straightforward and requires only a slight modification in the argument that follows; thus, it is excluded for brevity).

The introduction of dimensionless coordinates $\eta_{i}$ 's that immobilize the moving boundaries eliminates the difficulty in handling the unknown positions of the moving boundaries

$$
\eta_{i}=\frac{x-\hat{X}_{i-1}}{\delta_{i}}, \quad i=1,2,3, \ldots, N .
$$

Since $\hat{X}_{i-1} \leqslant x \leqslant \hat{X}_{i}$ in phase $i$ and $\delta_{i}=\hat{X}_{i}-\hat{X}_{i-1}$, each phase is then characterized by

$$
\text { phase } i: \quad 0 \leqslant \eta_{i} \leqslant 1, \quad i=1,2,3, \ldots, N
$$

for all time. A rigorous argument about the validity of the equalities in equation (7) is insignificant in the solution method and thus omitted.

Without sacrificing mathematical simplicity, the transformation of the governing equations (1) and (2) gives [20]

$$
\frac{\hat{c}}{\hat{c} t}\left(\rho_{i} \frac{\hat{c} V_{i}}{\hat{c} \eta_{i}}\right)+\frac{\partial F_{i}}{\hat{\partial} \eta_{i}}=0
$$

$$
\begin{gathered}
\frac{\hat{c}}{\hat{c} t}\left(\rho_{i} h_{i} \frac{\partial V_{i}}{\partial \eta_{i}}\right)+\frac{\partial J_{i}}{\partial \eta_{i}}=S_{i}\left(\frac{\partial V_{i}}{\hat{\partial} \eta_{i}}\right) \\
F_{i}\left(\eta_{i}, t\right)=\rho_{i}\left(f_{i}-\frac{\hat{c} V_{i}}{\hat{c} t}\right), \\
J_{i}\left(\eta_{i}, t\right)=F_{i} h_{i}-\frac{X_{i}^{n} k_{i}}{\delta_{i}} \frac{\partial T_{i}}{\partial \eta_{i}} \\
V_{i}\left(\eta_{i}, t\right)=\frac{1}{n+1}\left\{X_{i}\left(\eta_{i}, t\right)\right\}^{n+1}, \\
X_{i}\left(\eta_{i}, t\right)=\eta_{i} \delta_{i}(t)+\hat{X}_{i-1}(t)
\end{gathered}
$$

where $1 \leqslant i \leqslant N$. Note that the value of $X_{i}$ is the same as that of the coordinate $x$ so that $\hat{X}_{i-1} \leqslant X_{i} \leqslant \hat{X}_{i}$. Note also that the transformed equations degenerate to the original governing equations unless the positions of the boundaries change with time and that the conservative forms are preserved in the present transformation (the advantage of the conservative forms is that the conservation principle is obeyed exactly in each phase when a finite control-volume integration method is used to derive discretization equations [18]). After integration over time and over

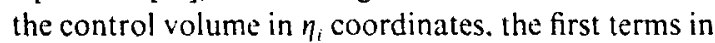
equations (8) and (9) denote the net change of the mass and the energy, respectively, contained in that control volume. The total mass flux $F_{i}$ combines the pseudo-convection terms created by the immobilization of the moving interfaces [12] and the convective terms due to physical motions of adjacent phases (which, if present, are caused by density variation between phases). The total heat flux $J_{i}$ represents both the diffusional heat flow rates and the net convective enthalpy How rates.

The interface boundary conditions, equations (4) and (5), are then expressed as

$$
\begin{gathered}
F_{m}=F_{m+1} \equiv \hat{F}_{m} \\
J_{m}=J_{m+1}
\end{gathered}
$$

where all values are evaluated at $\hat{X}_{m}$ (i.e. at $\eta_{m}=1$ and $\eta_{m+1}=0$ ), and the mass flow rate across the interface $\hat{X}_{m}$ is defined as $\hat{F}_{m}$ for future use. Note that the interfacial mass and energy balances are no more than the conditions of continuity of the flux terms in the transformed governing equations.

\section{SOLUTION PROCEDURE}

Before the solution method is presented. the role played by the interfacial mass balance in acquiring the velocity fields is stated briefly. The interfacial mass balance (12) can be rephrased as

$$
\begin{aligned}
\frac{\mathrm{d} \hat{V}_{m}}{\mathrm{~d} t}=f_{m}-\frac{\hat{F}_{m}}{\rho_{m}}=f_{m+1}-\frac{\hat{F}_{m}}{\rho_{m+1}}, \text { where } & \\
\hat{V}_{m} & =\frac{1}{n+1} \hat{X}_{m}^{n+1} .
\end{aligned}
$$

In general, there is at least one phase the value $f_{i}$ of 


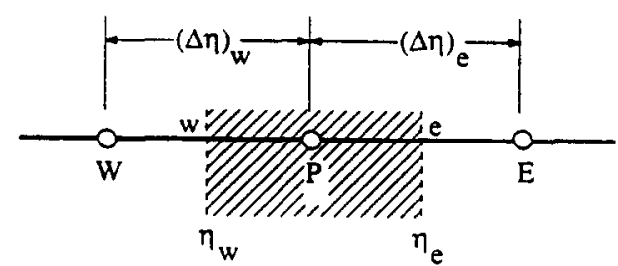

FIG. 2. Numerical grids and control volume faces for onedimensional problems.

which is known for all time (usually such a phase is stagnant, i.e. $f_{i}=0$ ) ; let it be phase $m$ without a loss of generality. All other values of $f_{i}$ can then be determined successively from the above equation starting with $f_{m}$ if all $\hat{F}_{i}$ obtained from the interfacial energy balances are prescribed.

The transformed conservation equations (8) and (9) are similar to those of the diffusion/convection problems (without phase change) discussed in the work of Patankar [18] and can be solved by an implicit method based on the finite control-volume integration procedure described in ref. [18]. The positions of the control volume faces are deployed in each phase according to the relation

$$
\eta_{i, j}=\left(\frac{j-1}{M_{i}-1}\right)^{p_{i}}, j=1,2,3, \ldots, M_{i}
$$

where $M_{i}$ is the total number of control volume faces in phase $i$, and $p_{i}$ is an exponent suited to the problem. The grid points are placed midway between the control volume faces so that a total of $\left(M_{i}-1\right)$ grid points are distributed inside each phase. Since the transformed conservation equations (8) and (9) have the same forms for different phases except the phase index $i$, discretization equations are derived only for phase $i$ yet applicable to other phases. For a typical control volume, shown in Fig. 2, the integration of equation (9) with the aid of equation (8) gives (for convenience. constant properties are assumed and the subscript $i$ is dropped except in $\delta_{i}$ and $\hat{X}_{i-1}$ )

$$
a_{\mathrm{P}} T_{\mathrm{P}}=a_{\mathrm{E}} T_{\mathrm{E}}+a_{\mathrm{W}} T_{\mathrm{W}}+b
$$

where

$$
\begin{gathered}
a_{\mathrm{p}}=a_{\mathrm{F}}+a_{\mathrm{W}}+a_{\mathrm{P}}^{0}-S_{\mathrm{P}}(\Delta V)_{\mathrm{P}}, \quad a_{\mathrm{P}}^{0}=\frac{\rho c(\Delta V)_{\mathrm{P}}^{0}}{\Delta t} \\
a_{\mathrm{E}}=D_{\mathrm{e}} A\left(\left|P_{\mathrm{e}}\right|\right)+\max \dagger\left\{-c F_{\mathrm{e}}, 0\right\} \\
a_{\mathrm{w}}=D_{\mathrm{w}} A\left(\left|P_{\mathrm{w}}\right|\right)+\max \left\{c F_{\mathrm{w}}, 0\right\} \\
D_{\mathrm{c}}=\frac{X_{\mathrm{c}}^{n} k}{\delta_{i}(\Delta \eta)_{\mathrm{e}}}, \quad D_{\mathrm{w}}=\frac{X_{\mathrm{w}}^{n} k}{\delta_{i}(\Delta \eta)_{\mathrm{w}}}, P_{\mathrm{e}}=\frac{c F_{\mathrm{c}}}{D_{\mathrm{e}}}, P_{\mathrm{w}}=\frac{c F_{\mathrm{w}}}{D_{\mathrm{w}}} \\
b=S_{\mathrm{C}}(\Delta V)_{\mathrm{P}}+a_{\mathrm{P}}^{0} T_{\mathrm{P}}^{0}, \quad(\Delta V)_{\mathrm{p}}=V_{\mathrm{c}}-V_{\mathrm{w}} \\
V_{\mathrm{e}}=\frac{1}{n+1} X_{\mathrm{c}}^{n+1}, \quad V_{\mathrm{w}}=\frac{1}{n+1} X_{\mathrm{w}}^{n+1} \\
X_{\mathrm{c}}=\eta_{\mathrm{c}} \delta_{i}+\hat{X}_{i-1}, \quad X_{\mathrm{w}}=\eta_{\mathrm{w}} \delta_{i}+\hat{X}_{i-1} .
\end{gathered}
$$

$\dagger \max \{A, B\}$ denotes the greater of $A$ and $B$
Table 1. Function $A(|P|)$ for different schemes [18]

\begin{tabular}{cc}
\hline Scheme & Formula for $A(|P|)$ \\
\hline $\begin{array}{c}\text { Central difference } \\
\text { Upwind } \\
\text { Hybrid } \\
\text { Power law }\end{array}$ & $1-0.5|P|$ \\
\hline
\end{tabular}

Here, the known values at time $t_{0}$ (i.e. the values at the beginning of the time step) are denoted by using the superscript 0 . In accordance with the implicit difference, all other values (i.e. without a superscript) are to be determined; $S_{\mathrm{C}}$ and $S_{\mathrm{p}}$ arise from the linearization of the source terms [18] ; and $D$ and $P$ (with the subscripts dropped) indicate the diffusion conductances and Peclet numbers, respectively. The function $A(|P|)$ can be selected from Table 1 (the same as Table 5.2 in ref. [18]) for the desired scheme. Nonconstant values of thermal conductivity and specific heat can be handled in the same way as in ref. [18].

It is interesting to note that, although the transformed equations are integrated over the transformed coordinates, all the quantities in the resulting dis. cretization equations have exactly their own physical interpretation. For example, $(\Delta V)_{\mathrm{P}}^{0}$ corresponds to the actual volume enclosed by two adjacent control volume faces at time $t_{0}$. The fact that $(\Delta V)_{\mathrm{P}}$ changes with time represents in effect the stretching/ contraction of the moving control volume in the physical coordinates which corresponds to the fixed control volume in the transformed coordinates.

The interfacial energy balance at $\hat{X}_{m}$. or equation (13), is discretized as

$$
\begin{aligned}
& \hat{F}_{m} h_{m}\left(\hat{T}_{m}\right)+\left[D_{m} A\left(\left|P_{m}\right|\right)+\max \left\{c_{m} \hat{F}_{m}, 0\right\}\right] \\
& \times\left(T_{m, \mathrm{~B}}-\hat{T}_{m}\right)=\hat{F}_{m} h_{m+1}\left(\hat{T}_{m}\right)+\left[D_{m+1} A\left(\left|P_{m+1}\right|\right)\right. \\
&\left.+\max \left\{-c_{m+1} \hat{F}_{m}, 0\right\}\right]\left(\hat{T}_{m}-T_{m+1 . \mathrm{B}}\right)
\end{aligned}
$$

where

$$
\begin{gathered}
D_{m}=\frac{\hat{X}_{m}^{n} k_{m}}{\delta_{m}(\Delta \eta)_{m}}, \quad D_{m+1}=\frac{\hat{X}_{m}^{n} k_{m-1}}{\delta_{m+1}(\Delta \eta)_{m+1}} \\
P_{m}=\frac{c_{m} \hat{F}_{m}}{D_{m}}, \quad P_{m+1}=\frac{c_{m+1} \hat{F}_{m}}{D_{m+1}} .
\end{gathered}
$$

The indices $m$ and $(m+1)$ represent the phases adjacent to the interface; $h_{m}\left(\hat{T}_{m}\right)$ and $h_{m+1}\left(\hat{T}_{m}\right)$ are the specific enthalpies of phase $m$ and phase $(m+1)$ at the phase temperature $\hat{T}_{m} ; T_{m . \mathrm{B}}$ and $T_{m+1 . \mathrm{B}}$ are the temperatures of the neighboring grid points to the interface $\hat{X}_{m}$, as shown in Fig. 3 ; and $(\Delta \eta)_{m}$ and $(\Delta \eta)_{m+1}$ are the distances between the interface $\hat{X}_{m}$ and the adjacent grid points. Equation (17) provides an implicit expression for $\hat{F}_{m}$ : in the case of a central difference scheme 


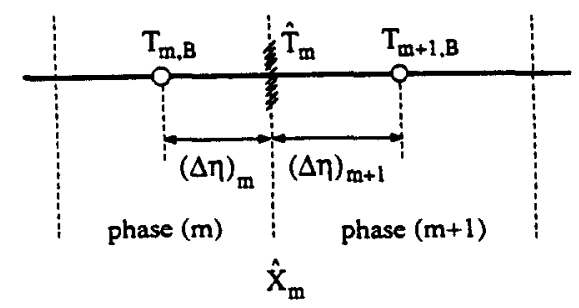

Fiv. 3. Grid-point cluster near the interface $\hat{X}_{m}$ at phase temperature $\dot{T}_{m}$.

$$
\hat{F}_{m}=\frac{D_{m}\left(T_{m, \mathrm{~B}}-\hat{T}_{m}\right)+D_{m+1}\left(T_{m+1 . \mathrm{B}}-\hat{T}_{m}\right)}{L_{m}-0.5 c_{m}\left(T_{m . \mathrm{B}}-\hat{T}_{m}\right)+0.5 c_{m+1}\left(T_{m+1 . \mathrm{B}}-\hat{T}_{m}\right)}
$$

where $L_{m}=h_{m+1}\left(\hat{T}_{m}\right)-h_{m}\left(\hat{T}_{m}\right)$, and the absolute value of $L_{m}$ represents the latent heat. $\hat{F}_{m}$ can also be obtained algebraically for other numerical schemes while trial and error is necessary for a power law scheme.

Let us suppose that all additional boundary conditions are specified for a particular problem, in addition to the phase interface conditions discussed previously. In solving the set of finite-difference equations (16) by a tridiagonal matrix algorithm, the unknown quantitics $\hat{X}_{i}$ 's and $F_{i}$ 's are required as input (values of $X_{i}^{\prime}$ 's, $V_{i}$ 's and $\delta_{i}$ 's can be derived from $\hat{X}_{i}^{\prime}$ 's). While $\hat{X}_{i}$ 's are evaluated at time $\left(t_{0}+\Delta t\right)$ in accordance with the implicit difference, $F_{i}$ 's are evaluated at time $\left(t_{0}+\frac{1}{\Delta} t\right)$ (by interpreting an implicit difference as a central difference representation with respect to time $\left(t_{0}+[\Delta t)[11]\right)$ and discretized as

$$
F_{i}^{1 \cdot 2}=\rho_{i}\left\{f_{i}^{1 / 2}-\left(\frac{\partial V_{i}}{\partial t}\right)^{1 / 2}\right\}=\rho_{i}\left(f_{i}^{1 / 2}-\frac{V_{i}-V_{i}^{0}}{\Delta t}\right)
$$

where the superscript $1 / 2$ indicates the quantities at time $\left(t_{0}+\frac{1}{2} \Delta t\right)$. Therefore, all $f_{i}^{1 / 2}$ s and $\hat{X}_{i}$ 's are required to determine the unknown temperature distributions $T_{i}$ 's at time $\left(t_{0}+\Delta t\right)$. The needed values can be obtained via the interfacial mass and energy balances as follows.

First, $\hat{F}_{m}^{0}$ is determined by evaluating the interfacial energy balance (17) at time $t_{0} .\left(\mathrm{d} \hat{V}_{m} / \mathrm{d} t\right)^{0}$ and $f_{m+1}^{0}$ are calculated from the interfacial mass balance (14) with the known values of $f_{m}^{0}, \hat{F}_{m}^{0}$. Next, $\hat{V}_{m}$ at time $\left(t_{0}+\frac{1}{2} \Delta t\right)$ is obtained as

$$
\hat{V}_{m}^{12}=\hat{V}_{m}^{0}+\left(\frac{\mathrm{d} \hat{V}_{m}}{\mathrm{~d} t}\right)^{0} \frac{\Delta t}{2}
$$

and $X_{m}^{1,2}$ then follows. It should be noted here that there can be boundaries for which the above procedure is unnecessary since $\hat{X}_{i}(t)$ 's may already be assigned, depending on the problem. For example, $\hat{X}_{0}$ indicates the origin of the system and remains unchanged with time (i.e. $\hat{X}_{0}(t)=0$ ), as shown in Fig. 1. Thus, all values of $\hat{X}_{i}^{1 / 2}$ s can be determined by following the above procedure or by prescribing them. All $\delta_{i}^{1:}$ "s then follow immediately. Similarly, $\hat{F}_{m}^{1 / 2}$ is determined by updating $\hat{X}_{i}$ 's and $\delta_{i}$ 's in equation (17) with the quantities at time $\left(t_{0}+\frac{1}{\Delta} \Delta t\right)$, except the temperature fields. In addition, $\left(\mathrm{d} \hat{V}_{m} / \mathrm{d} t\right)^{1 / 2}$ and $f_{m+1}^{1,2}$ are obtained from equation (14). Finally, $\hat{V}_{m}$ and $\hat{X}_{m}$ at time $\left(t_{0}+\Delta t\right)$ are determined as

$$
\hat{V}_{m}=\hat{V}_{m}^{n}+\left(\frac{\mathrm{d} \hat{V}_{m}}{\mathrm{~d} t}\right)^{1} \Delta t, \quad \hat{X}_{m}=\left\{(n+1) \hat{V}_{m}\right\}^{1 \cdot(n+1)} .
$$

It then becomes possible to solve the unknown temperature fields $T_{i}$ 's at time $\left(t_{0}+\Delta t\right)$ with known values of $\hat{X}_{i}$ 's and $f_{i}^{\prime}$ 's, provided the additional boundary conditions corresponding to a specific problem are completely elucidated.

The advantage of this approach is that it enables the solution to march steadily forward in time without requiring iterations at each time step while the positions of the interfaces are being updated [12]. The explicit treatment of the interfacial conditions can still assure the conservation of mass fluxes over all phases. Although the heat fluxes are also conserved within each phase by utilizing the present coordinate transformation, the heat fluxes at the interfaces may be inconsistent due to the explicit treatment of the moving boundaries. Those inconsistencics are adjusted by updating the positions of the interfaces; thus, the generation of parasitical heat sources is forced to vanish at each time step, which assures the overall energy conservation within a tolerance.

\section{EXAMPLE PROBLEMS}

The accuracy and flexibility of the present numerical method are examined in this section for the case of 1-D geometry. Particular 1-D phase-change problems are solved and compared with existing analytical, semi-analytical and numerical solutions.

\subsection{Neumann problem with volume changes}

Consider the solidification of a liquid in a semiinfinite plane for which phases 1 and 2 are the solid and the liquid phases, respectively; $\hat{X}_{1}$ is the solid/ liquid interface with $L_{1}=h_{\mathrm{s}}$; and $\hat{X}_{2}$ is the front of a thermal boundary layer that diffuses into the liquid phase starting at the interface $\hat{X}_{1}$. The additional boundary conditions are

$$
\begin{gathered}
T_{1}(0, t)=\hat{T}_{0}, \quad T_{2}(1, t)=T_{2}\left(\eta_{2}, 0\right)=\hat{T}_{2}, \\
\delta_{1}(0)=0 \quad\left(\hat{T}_{0}<\hat{T}_{1} \leqslant \hat{T}_{2}\right) .
\end{gathered}
$$

To illustrate the volumetric effects, consider the case of $\rho_{1} \geqslant \rho_{2}$. The dimensionless parameters are

$$
\frac{\alpha_{2}}{\alpha_{1}}, \frac{k_{2}}{k_{1}}, \rho=\frac{\rho_{2}}{\rho_{1}}, \phi=\frac{\hat{T}_{2}-\hat{T}_{1}}{\hat{T}_{1}-\hat{T}_{0}}, \text { Ste }=\frac{c_{1}\left(\hat{T}_{1}-\hat{T}_{0}\right)}{L_{1}}
$$


where $\rho$ is a density ratio; $\phi$ a superheating parameter; and Ste the Stefan number, which is a measure of sensible heat to latent heat. Assume that $k_{1}=k_{2}$ and $x_{1}=x_{2}$ to reduce the number of parameters. The exact solution shows that

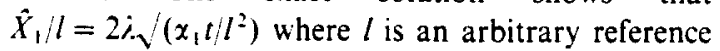
length, and $i$ is a similarity constant obtained by the following equation [1]:

$$
\frac{i \sqrt{ } \pi}{S t e}=\frac{\mathrm{e}^{-\lambda^{2}}}{\operatorname{erf} i}-\frac{\phi \mathrm{e}^{-\lambda^{2} \rho^{2}}}{\operatorname{erfc}(\lambda / \rho)}
$$

For a more sensitive test of the quality of the numerical method, the positions of the phase interface with time are chosen as the compared quantities. Therefore, the value of $\lambda$ obtained numerically will be compared with that from the above equation for the same values of $\rho, \phi$, and Ste.

A value of $\hat{X}_{2}$ is selected to be large enough so that grid points near $\hat{X}_{2}$ remain thermally dormant and do not affect the solution. One simple way to achieve this objective is to assume that $\hat{X}_{2}=C \hat{X}_{1}$, where $C$ is a constant factor [12]. Numerical parameters defined in equation (15) are set to be $M_{1}=51, M_{2}=301$, $p_{1}=1.0, p_{2}=1.1$, and $C=500$. The assumption that a solid layer of thickness $\delta_{1} / l=10^{-6}$ exists at time $t=0$ avoids start-up difficulties due to the singularity in the initial condition. A linear temperature profile is prescribed in the solid phase (considering the thinness of the solid layer), while the temperatures of the liquid phase are initialized with $\hat{T}_{2}$. The time steps are selected so that the maximum change in $\delta_{i}$ is less than $2 \%$ at each time step. The effect of this initially prescribed solid layer upon the subsequent results vanishes in small elapsed times, and the numerical solutions nearly follow the exact ones before $\delta_{1} / l$ reaches, at most, a value of $10^{-5}$ for a wide range of parameters. Therefore, there is no need to start the calculation with the analytic solution as far as a small time solution is not the main concern. Thus, calculations continue until $\delta_{1} / l=100$.

Table 2 shows the maximum percentage errors between the results for a similarity constant $\lambda$ from the exact solution (equation (24)) and the present numerical method during the interval $10^{-4}<\delta_{1} / l<10^{2}$. Excellent agreement with the exact solutions over a wide range of parameters is evident in Table 2. The results also show the effect of various schemes on the accuracy. Although Ste $<5$ represents the range of interest in most real situations, high Stefan numbers are included to show the performance of various numerical schemes more clearly. The upwind scheme gives the least accurate results, while negligible differences are observed in the results obtained by employing other schemes. The efficiency of the power law scheme deteriorates due to the additional work needed to find roots by trial and error (see equation (17)). In general, both central difference and hybrid schemes yield good results in addition to providing simple solutions to the interfacial energy balance (in most phase-change problems, the interface moves relatively slow, therefore allowing the use of the central difference scheme [16]). For this reason, the central difference scheme is employed to solve the subsequent examples.

\subsection{Bubble grow th problem}

The growth of a spherical bubble in a superheated liquid is considered next so the effects of system geometry (or $n=2$ ) can be examined. In this system. phases 1 and 2 are the gas and the liquid phases, respectively; $\hat{X}_{1}$ is the gas liquid interface with $L_{1}=-h_{\mathrm{ig}} ;$ and $\hat{X}_{2}$ is the front of a thermal boundary layer. In addition

$$
\begin{gathered}
T_{1}\left(\eta_{1}, t\right)=\hat{T}_{1}, \quad T_{2}\left(\eta_{2}, 0\right)=\hat{T}_{2}, \quad \hat{X}_{1}(0)=0 . \\
T_{2}(1, t)=\hat{T}_{2}>\hat{T}_{1}
\end{gathered}
$$

with the dimensionless parameters

$$
\rho=\frac{\rho_{2}}{\rho_{1}}, \quad \text { Ste }=\frac{\rho_{2}}{\rho_{1}} \frac{c_{2}\left(\hat{T}_{2}-\hat{T}_{1}\right)}{-L_{1}} .
$$

The analytic solution by Scriven [21] shows that $\hat{X}_{1} / l=2 \lambda \sqrt{ }\left(\alpha_{2} t / l^{2}\right)$ where the relation between Ste

\begin{tabular}{|c|c|c|c|c|c|c|c|}
\hline Ste & $\rho$ & $\phi$ & $i$ & Central & Upwind & Hybrid & Power law \\
\hline \multirow[t]{4}{*}{0.01} & 1 & 0 & 0.07059 & -0.01 & 0.00 & -0.01 & -0.01 \\
\hline & & 5 & 0.05719 & -0.09 & -0.38 & -0.09 & -0.10 \\
\hline & 0.5 & I & 0.06742 & -0.03 & -0.10 & -0.03 & -0.03 \\
\hline & & 5 & 0.05646 & -0.10 & $-0.4 !$ & -0.10 & -0.11 \\
\hline \multirow[t]{4}{*}{1} & 1 & 0 & 0.62006 & -0.10 & 0.11 & -0.10 & -0.10 \\
\hline & & 5 & 0.14351 & -0.13 & -2.68 & -0.13 & -0.26 \\
\hline & 0.5 & 1 & 0.33505 & 0.49 & -2.30 & 0.49 & 0.08 \\
\hline & & 5 & 0.12936 & -0.19 & -2.47 & -0.19 & -0.31 \\
\hline \multirow[t]{4}{*}{100} & 1 & 0 & 1.85095 & -0.37 & 1.73 & -0.37 & -0.33 \\
\hline & & 5 & 0.14874 & -1.49 & -5.58 & -1.97 & -4.42 \\
\hline & 0.5 & 1 & 0.39310 & 1.00 & -3.20 & 1.00 & 0.28 \\
\hline & & 5 & 0.13287 & -1.08 & -5.15 & -1.11 & -1.96 \\
\hline
\end{tabular}
and $i$ is given as

Table 2. Percentage error in values of $\lambda$ 's for various schemes (compared with the exact values of $i$ 's in equation (24))

$\operatorname{Error}(\%)=\left(\left(\lambda_{\text {exact }}-\lambda_{\text {numerical }}\right) / \lambda_{\text {exact }}\right) \times 100$. 


$$
\begin{aligned}
\text { Ste }=2 \dot{\int_{i}} \frac{i^{2}}{x^{2}} \exp & \left\{i^{2}-x^{2}\right. \\
& \left.+2 i^{3}\left(1-\frac{1}{\rho}\right)\left(\frac{1}{i}-\frac{1}{x}\right)\right\} \mathrm{d} x .
\end{aligned}
$$

The calculation starts with $\hat{X}_{1} / l=10^{-6}$ and a uniform temperature field in the liquid phase at time $t=0$. The edge of the thermal boundary layer $\hat{X}_{2}$ is carefully chosen so that it does not affect the heat transfer process at the interface $\hat{X}_{1}$, and it is allowed to increase with $\hat{X}_{1}$. The computations are performed with $M_{2}=101, p_{2}=2.5$. Table 3 shows the maximum percentage errors for $\lambda$ 's produced by the present numerical method during the interval $10^{-4}<\hat{X}_{1} / l<10^{2}$ and by the analytic solutions of equation (27). Good agreement exists between the solutions even for a large density ratio. Note that the effect of a density change becomes insignificant as Ste $\rightarrow 0$ for the Stefan numbers defined as above.

\subsection{Inward solidification problem}

The inward solidification of a saturated liquid in a sphere of radius $l$ has been treated by many investigators $[5,22,23]$ for either constant temperature or convection at the wall. The case with convection at the wall will be considered here, for which

$$
\begin{gathered}
T_{1}\left(\eta_{1}, t\right)=\hat{T}_{1}, \quad \delta_{2}(0)=0, \\
J_{2}(1, t)=X_{2}^{n} h_{x}\left\{T_{2}(1, t)-T_{x}\right\}
\end{gathered}
$$

where phases 1 and 2 are the liquid and the solid phases, respectively; $\hat{X}_{1}$ is the phase interface with $L_{1}=-h_{\mathrm{sf}}$; and $\hat{X}_{2}$ is the fixed wall (i.e. $\hat{X}_{2}(t)=l$ ). The dimensionless parameters are

$$
\text { Ste }=\frac{c_{2}\left(\hat{T}_{1}-T_{s}\right)}{-L_{1}}, \quad B i=\frac{h_{s} l}{k_{2}} .
$$

A linear temperature profile with a negligible temperature drop is prescribed within the solid layer of thickness $\delta_{2} / l=10^{-6}$ at time $t=0$, which avoids start-up difficulties. An overall energy balance is taken from the instant freezing begins (i.e. $t=0$ ) to each time step

$$
\int_{0}^{t} x^{\prime \prime} \rho\left(h-h^{*}\right) \mathrm{d} x+\int_{0}^{t} J_{2}(1, t) \mathrm{d} t=\text { constant }
$$

Table 3. Maximum percentage errors for values of $i$ compared with $\lambda$ 's in equation (27)

\begin{tabular}{cccc}
\hline$\rho$ & $i$ & Ste & Error $(\%)$ \\
\hline 1 & 0.01 & $1.965 \times 10^{-4}$ & -0.40 \\
& 0.1 & $1.682 \times 10^{-2}$ & -0.12 \\
& 1 & 0.4843 & -0.63 \\
1000 & 0.01 & $1.965 \times 10^{-4}$ & -0.40 \\
& 0.1 & $1.697 \times 10^{-2}$ & -0.11 \\
& 1 & 0.6975 & -0.26 \\
\hline
\end{tabular}

$\operatorname{Error}(\%)=\left(\left(\lambda_{\text {exact }}-\lambda_{\text {numerical }}\right) / \lambda_{\text {exact }}\right) \times 100$. which is useful in assessing the validity of the numerical solutions in terms of the physical reality. The above overall energy balance is checked at every time step to determine if it is satisfied within a tolerance (say, $0.1 \%$ for the present example with the reference enthalpy $h^{*}$ being that of the solid phase at its fusion temperature).

Figure 4 shows the timewise variation of $\bar{X}_{1}(t)$ for Ste $=0.5$ and three Biot numbers. The values of $\hat{X}_{1}$ with time are sufficiently close to those of refs. [22, 23], while some discrepancies are noted as the freezing front approaches the center. The time for complete solidification of the sphere, $t_{\mathrm{c}}$, is assumed to be equal to a time at which $\hat{X}, / l$ reaches a value of $10^{-3}$. Table 4 compares the results for the dimensionless freezing time $\tau_{\mathrm{c}}\left(=x_{2} t_{\mathrm{c}} / l^{2}\right)$ from the approximate analytic method [22] with those obtained by the present numerical method. The terms $\tau_{1 \mathrm{c}}, \tau_{2 \mathrm{c}}$, and $\tau_{i \mathrm{i}}$ mean the solidification time $\tau_{c}$ by evaluating the first one, two, and three terms of the approximate analytic solutions [22], respectively. The disagreement with the values from ref. [22] becomes significant with increasing Stefan numbers, while good agreement is noted for Ste $\leqslant 0.1$. This failure probably arises because the convergence of the estimates $\tau_{\mathrm{k},}, \tau_{\mathrm{z}}$, and $\tau_{\mathrm{k}}$ from ref. [22] becomes weaker with increasing Ste. since only the first three terms of the approximate analytic solulions are considered.

It is then natural to expect the degree of agreement to improve if more than the first three terms of the approximate analytic solutions can be included. An attempt to include more terms was made for the planar case $(n=0)$ by Pedroso and Domoto [6], who developed the exact solutions for the case of Ste $\leqslant 1$ by including as many terms as desired in their perturbation technique (actually, the first nine terms were considered). For this planar case $(n=0)$, the solutions of the dimensionless freezing time for $B i=1$, and Ste $=0.5$ and 1.0 give $(B i)^{2}(S t e) \tau_{\mathrm{c}}=1.656$ and 1.796 , respectively, while the corresponding solutions from ref. [6] are $(B i)^{2}(S t e) \tau_{\mathrm{c}}=1.656$ and 1.804 . respectively. The difference between these two results is negligible, as expected.

\subsection{Phase change with heat generation}

Freezing and melting in a heat-generating. 1-D slab of thickness $2 l$ is chosen to test the effects of heat generation. The 1-D slab is initially molten and trapped between two semi-infinite walls at temperatures below the freezing point of the slab. This problem was originally studied numerically by Cheung et al. [13] using the method of collocation. Only one-half of the system is considered. since the heat transfer process is assumed to be symmetrical with respect to the centerline of the slab, which is taken to be the origin of the system. Here. phases 1 and 2 are the liquid and the solid phases of a heatgenerating substance with a uniform heat source $S_{2}\left(=S_{1}\right)$, respectively, and phase 3 is a cold wall with $S_{3}=0 ; \hat{X}_{1}$ is the liquid/solid interface with $L_{1}=$ 


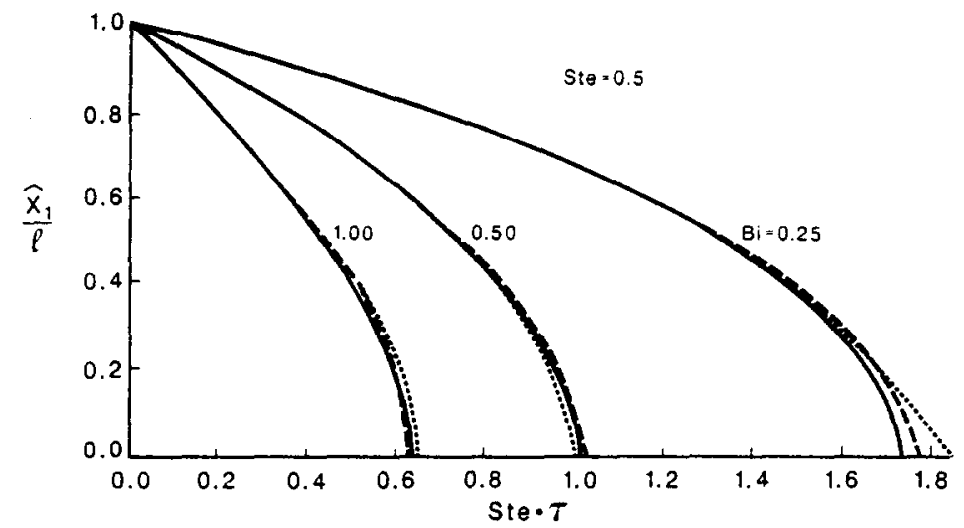

FIG. 4. Timewise variation of the freezing front. This work, Tao [23].

Table 4. Dimensionless freezing time of a sphere with $B i=0.2$ (compared with the results from ref. [22])

\begin{tabular}{lcccc}
\hline Stc & $\tau_{1 \mathrm{c}}$ & $\tau_{3 \mathrm{~s}}$ & $\tau_{\mathrm{k}}$ & $\tau_{\mathrm{c}}$ \\
\hline 2.0 & 1.55 & 1.42 & 1.17 & 1.26 \\
1.0 & 2.53 & 2.55 & 2.38 & 2.25 \\
0.5 & 4.40 & 4.51 & 4.47 & 4.17 \\
0.2 & 9.92 & 10.09 & 10.17 & 9.81 \\
0.1 & 19.09 & 19.28 & 19.41 & 19.08 \\
0.01 & 184.1 & 184.3 & 184.5 & 184.5 \\
\hline
\end{tabular}

$-h_{\mathrm{s} r} ; \quad \hat{X}_{2}$ is the fixed boundary (or $\hat{X}_{2}(t)=l$ ) separating two substances; and $\hat{X}_{3}$ is the edge of a thermal boundary layer in the cold wall. The additional boundary conditions are

$$
\begin{gathered}
T_{1}\left(\eta_{1}, 0\right)=\hat{T}_{0}, \quad T_{3}\left(\eta_{3}, 0\right)=T_{3}, \\
\delta_{2}(0)=0 \quad\left(\hat{T}_{0} \geqslant \hat{T}_{1}>\hat{T}_{3}\right) \\
\frac{\partial T_{1}}{\partial \eta_{1}}(0, t)=0, \quad T_{2}(1, t)=T_{3}(0, t), \\
J_{2}(1, t)=J_{3}(0, t) . \quad T_{3}(1, t)=\dot{T}_{3} .
\end{gathered}
$$

For brevity, the present calculations are carried out when $0<\delta_{2}<l$, for which the above system of equations is valid (the details are described in ref. [13]).

The thermophysical properties of a heat-generating substance are assumed to be the same in both phases and the dimensionless parameters are then

$$
\begin{gathered}
\frac{k_{3}}{k_{2}}, \frac{\alpha_{3}}{\alpha_{2}}, \quad A=\frac{S_{2} l^{2}}{k_{2}\left(\hat{T}_{1}-\hat{T}_{3}\right)}, \\
\phi=\frac{\hat{T}_{0}-\hat{T}_{3}}{\hat{T}_{1}-\hat{T}_{3}}(\geqslant 1), \quad \text { Ste }=\frac{c_{2}\left(\hat{T}_{1}-\hat{T}_{3}\right)}{-L_{1}}
\end{gathered}
$$

$\dagger$ It seems reasonable to neglect the differences contributed by the region $x: l<-2$, because the two profiles are nearly identical in this region. where the case of $k_{3} / k_{2}=x_{3} / x_{2}$ is considered, which simplifies the presentation of the numerical results. The initial singularity is avoided following a similar procedure to that discussed previously, and special care is taken for values of $\hat{X}_{3}$, which are selected continuously to be large enough so the results are not affected. By the nature of the problem. the results should satisfy the following relation:

$$
\int_{0}^{x} \rho\left(h-h^{*}\right) \mathrm{d} x-S / t=\text { constant }
$$

which represents the overall energy balance reflecting the adiabatic conditions at the centerline of the slab and at infinity, and the contribution of the uniform heat generation. The above condition proves to be valid within a tolerance of $0.1 \%$ throughout the results presented below (with $h^{*}$ being that of $h_{2}$ evaluated at $\hat{T}_{3}$ ).

Figure 5 shows the dimensionless temperatures vs the physical distance normalized by the half-width of the slab for the case of $A=1, \phi=1$. Ste $=2$, and $k_{3} / k_{2}=1$ where the dimensionless time $\tau\left(=x_{2} t / l^{2}\right)$ is used. The dimensionless thickness of the solid layer, $\delta_{2} / l$, attains a value of 0.224 at $\tau=0.05$ and achieves a maximum of 0.356 at $\tau=0.29$, while the corresponding solutions from ref. [13] give 0.23 at $\tau=0.05$, and a maximum of 0.34 at $\tau=0.3$, respectively. The temperature distributions agree well at the initial stage of freezing but deviate significantly as $\tau$ increases. The discrepancies can be explained with the aid of the overall energy balance mentioned above. The two profilest corresponding to $\tau=1.2$ show that the profile from ref. [13] is always beyond that of the present study over the ranges shown in Fig. 5. The energy associated with the temperature profiles from the present numerical method represents the sum of the energy initially contained in the system and the amount of energy generated and stored within the system until $t=1.2$ within a tolerance of $0.1 \%$. Therefore, the results from ref. [13] can probably be thought 


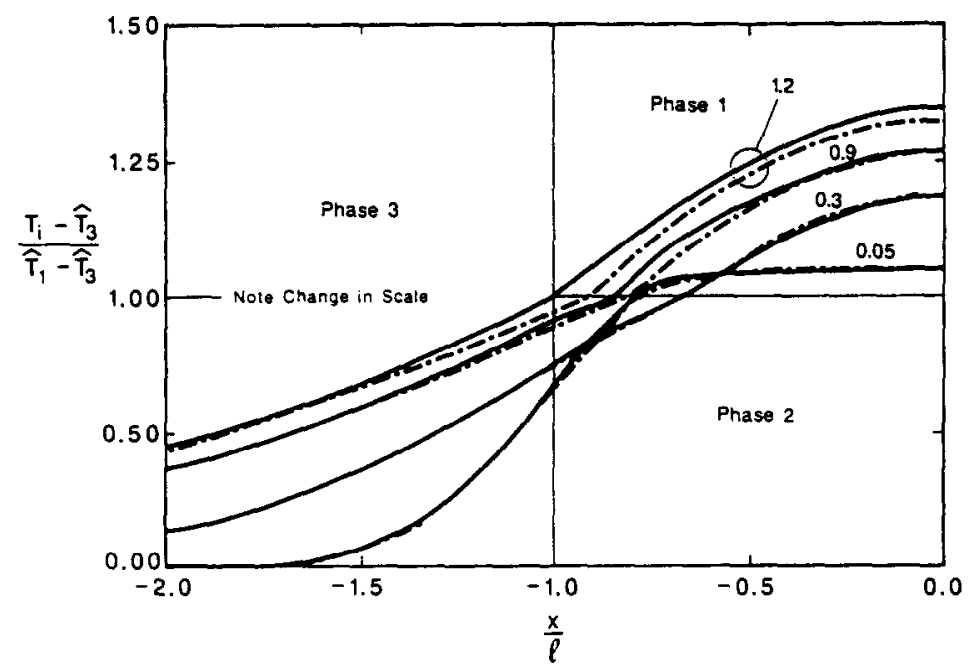

FiG. 5. Dimensionless temperature distribution of the system for the case of $A=1, \phi=1$, Ste $=2$, and $k_{3} / k_{2}=1$. This work, --- - C Cheung et al. [13]. -

to have created parasitical heat sources, and their effects on the temperature distribution become detectable at a large value of $\tau$.

This argument is consistent with the results shown in Fig. 6 which demonstrates the dependence of the transient solid layer thickness on the dimensionless heat generation for the case of $\phi=1$, Ste $=1$, and $k_{3} / k_{2}=1$. The lifetimes of the solid layers from ref. [13] are always significantly lower than those obtained by the present numerical method, thus it can be thought that the suspected additional heat sources in ref. [13] accelerate the decaying of the solid layer (except in the case of $A=0$, in which heat generation does not come into play).

The flexibility of the present numerical method is noteworthy when one explores the effect of system geometry further. The case of a heat-generating sphere for which only a steady solution is presented in ref. [13] can be resolved in the present study by merely changing the geometry index $n$. The behavior of the freezing front in a sphere, shown in Fig. 6. indicates that a heat-generating sphere can be cooled more easily than a heat-generating slab, as was predicted in ref. [13].

\section{EXTENSION TO MULTI-DIMENSIONAL PROBLEMS}

The numerical method formulated and tested for 1-D phase-change problems can be extended to solve multi-dimensional problems but 2-D cases (without density changes between phases) are considered in this section. In general, the $(x, y)$ rectangular coordinate and the $(x, \theta)$ cylindrical coordinate shown in Fig. 7 are transformed to new coordinates $\left(\eta_{i}, \vdots\right)$ where $\eta_{i}=\left(x-\hat{X}_{i-1}\right): \dot{\delta}_{i}$ and $\xi=y$ (or $\theta$ ). With the details omitted, the transformed conservation equations are

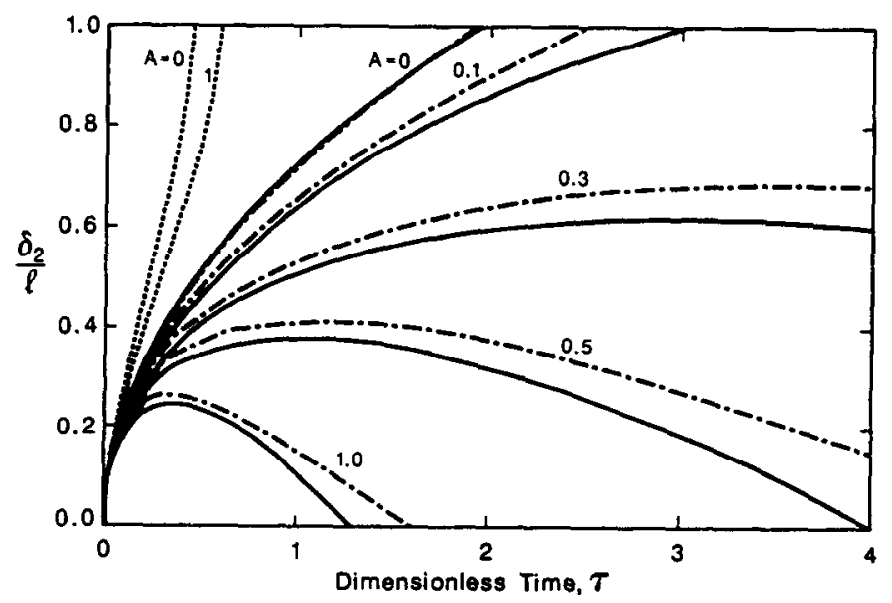

FIG. 6. Dimensionless positions of the freezing front with time for the case of $\phi=1$, Ste $=1$, and $k_{3} k_{2}=1$. This work, - - - (planar), ...- spherical; Cheung et al. [13], _- 


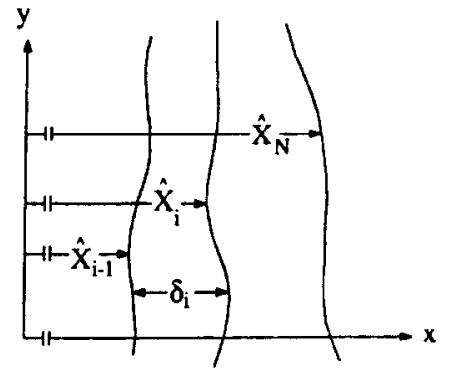

(a) the $(x, y)$ coordinate system

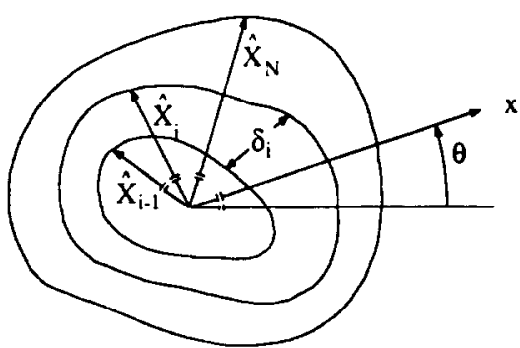

(b) the $(x, \theta)$ coordinate system

FIG. 7. Schematic of configuration for both rectangular and cylindrical coordinates-systems composed of $N$ phases.

$$
\begin{aligned}
& \frac{\hat{c}}{\hat{c} t}\left(\rho_{i} h_{i} \frac{\partial V_{i}}{\partial \eta_{i}}\right)+\frac{\partial J_{i}}{\hat{c} \eta_{i}}+\frac{\hat{c}}{\partial \xi}\left(-\frac{\delta_{i} k_{i}}{X_{i}^{\prime \prime}} \frac{\partial T_{i}}{\hat{c} \xi}\right) \\
& =S_{i} \frac{\hat{c} V_{i}}{\hat{c} \eta_{i}}-\frac{\hat{\partial}}{\hat{c} \eta_{i}}\left(\beta_{i} k_{i} \frac{\hat{c} T_{i}}{\hat{c} \xi}\right)-\frac{\hat{c}}{\hat{c} \xi}\left(\beta_{i} k_{i} \frac{\hat{c} T_{i}}{\hat{c} \eta_{i}}\right) \\
& F_{i}=-\rho_{i} \frac{\hat{\partial} V_{i}}{\hat{\imath} t}, \quad J_{i}=F_{i} h_{i}-\frac{X_{i}^{n} k_{i}}{\delta_{i}}\left(1+\beta_{i}^{2}\right) \frac{\partial T_{i}}{\partial \eta_{i}} \\
& \beta_{i}=\frac{1}{X_{i}^{n}} \frac{\hat{c} X_{i}}{\partial \xi}, V_{i}=\frac{1}{n+1} X_{i}^{n+1}, X_{i}=\eta_{i} \dot{\delta}_{i}+\hat{X}_{i-1}
\end{aligned}
$$

where $1 \leqslant i \leqslant N$ and $n=0$ for a rectangular coordinate, 1 for a cylindrical coordinate, respectively. The interfacial mass and energy balances are the same as equations (12) and (13). This can be justified by the fact that the heat flux in the $\eta_{i}$-direction (i.e. normal to the phase boundary) contributed by the sccond term on the right-hand side is zero because the phase boundary is an isotherm (thus $\partial T_{i} / \hat{c} \xi=0$ along the phase boundary). To obtain discretization equations from the above equations, a quite similar procedure. as discussed previously, is applied to the terms on the left-hand side. The treatment is implicit, while the quantities $\hat{X}_{i}$ 's (thus $\beta_{i}$ 's, $\delta_{i}$ 's, $V_{i}$ 's, and $X_{i}$ 's) can be obtained explicitly in the same way as before. The last two terms on the right-hand side are treated explicitly and a linear temperature profile is used to evaluate the gradients [24]. Also a piecewise linear profile is used to evaluate $\beta_{i}$.

As a test problem, consider an infinitely long cylinder of square cross-section, initially filled with liquid at its freezing temperature $\hat{T}_{1}$. At zero time, the surface temperature is lowered to $\hat{T}_{2}$ and maintained constant thereafter. Then the freezing front starts from the surface and moves inward until complete solidification is achieved. In this example. $\rho_{2}, c_{2}$, and $k_{2}$ are taken as constants and the dimensionless parameter is the Stefan number, Ste $=c_{2}\left(\hat{T}_{1}-\hat{T}_{2}\right) / h_{\mathrm{si}}$. Solutions are obtained only for a representative octant of the square due to symmetry and a $20 \times 20$ grid is used in the cylindrical coordinate (i.e. $n=1$ ). The symmetry lines are adiabatic and are characterized by $\beta_{2}=0$ and $\hat{r} T_{2} / \partial \zeta=0$. Figure 8 shows the position of the freezing front on the $x$-axis and its $x$-coordinate on the diagonal obtained by the present numerical method, where the $x$-axis is taken to be an adiabatic line opposite to the diagonal. The results obtained by Crank and Gupta [10]. Crowley [8], Allen and Severn [25] and Lazaridis [26] are also shown for comparison. The results of this study are in closer agreement with those of Crowley [8]. The numerical solution of the dimensionless time $\left(\tau=x_{2} t / l^{2}, l\right.$ is the half-width of the square) for complete solidification gives 0.626 while the corresponding solution from ref. [8] is $\sim 0.625$.

Next, the melting (or freezing) of a finite cylinder of height $H$ and radius $l$ is considered, which was studied originally by Duda et al. [15]. Only a brief description of the problem is given here and a sketch is shown in Fig. 9 (the details are given in ref. [15]). The cylinder contains both the solid and liquid phases the fusion temperature of which is $\hat{T}_{1}$, with the upper phase being solid and the lower phase liquid. Initially this system is at a steady-state condition with the upper surface of the cylinder maintained at a temperature $\hat{T}_{0}\left(<\hat{T}_{1}\right)$ and the lower surface at $\hat{T}_{2}\left(>\hat{T}_{1}\right)$ : the side surface is thermally insulated and the phase boundary is at $z=\dot{Z}_{1}^{0}$. At $t=0$ the insulation is removed and the side surface is exposed to a sur. rounding at $T_{x}\left(>\hat{T}_{1}\right)$ with the constant convective heat transfer coefficient $h_{x}$. Then the phase boundary, $\hat{Z}_{1}(t, r)$. starts to move upward and reaches eventually a steady-state position. For this problem, the $(r . z)$ coordinate is transformed into a new coordinate $(\zeta$. $\eta_{i}$ ) where $\zeta=r, \eta_{i}=\left(z-\hat{Z}_{i-1}\right) / \delta_{i}$ and the index $i=1$ refers to the upper phase, $i=2$ to the lower phase. respectively. The transformed governing equations for $T_{i}\left(t . \zeta, \eta_{i}\right)$ are

$$
\begin{aligned}
& \frac{\hat{c}}{\hat{c} t}\left(\rho_{i} h_{i} \zeta \frac{\hat{c} Z_{i}}{\hat{c} \eta_{i}}\right)+\frac{\hat{c} J_{i}}{\hat{\partial} \eta_{i}}+\frac{\hat{c}}{\hat{C} \xi}\left(-\xi \dot{\delta}_{i} k_{i} \frac{\hat{c} T_{i}}{\hat{C} \xi}\right)
\end{aligned}
$$

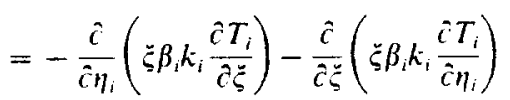




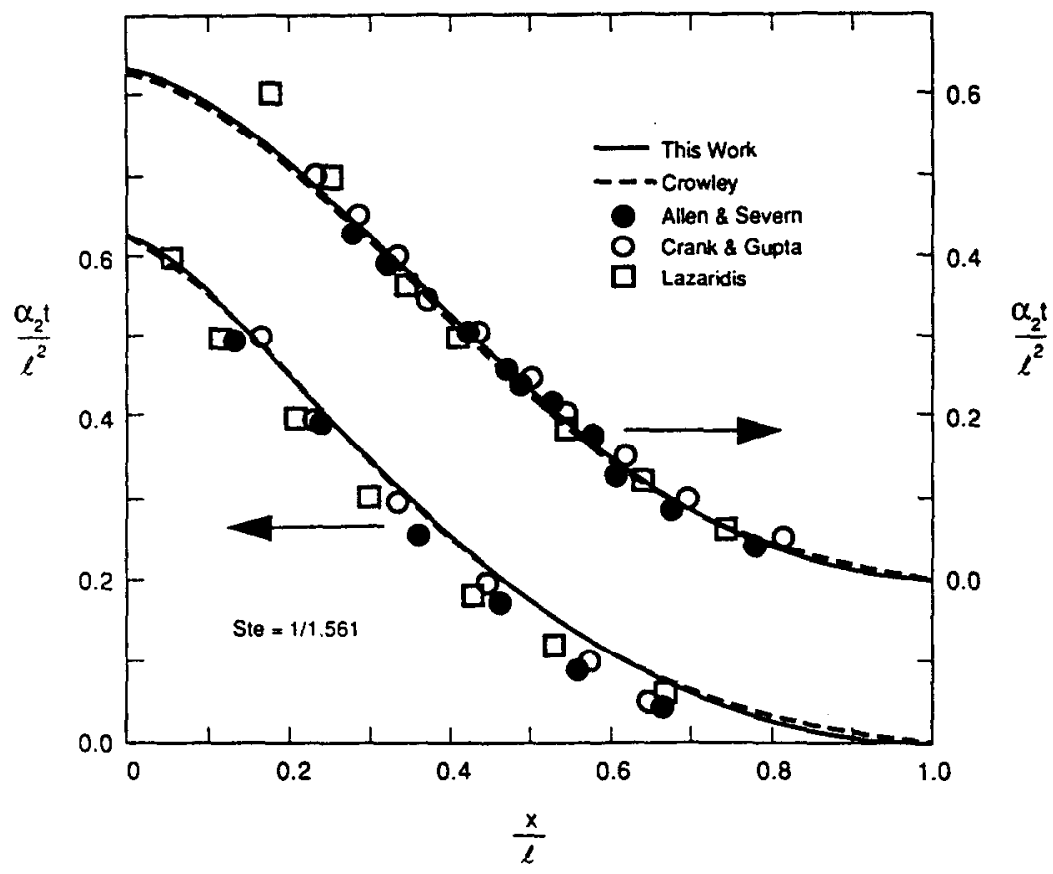

FIG. 8. The position of the freezing front on the $x$-axis (upper curve) and its $x$ coordinate on the diagonal (lower curve).

$$
\begin{gathered}
F_{i}=-\rho_{i} \xi \frac{\hat{\partial} Z_{i}}{\hat{\partial} t}, \quad J_{i}=F_{i} h_{i}-\frac{\xi k_{i}}{\delta_{i}}\left(1+\beta_{i}^{2}\right) \frac{\partial T_{i}}{\hat{c} \eta_{i}} \\
\beta_{i}=\frac{\partial Z_{i}}{\partial \grave{\zeta}}, \quad Z_{i}=\eta_{i} \delta_{i}+\hat{Z}_{i-1}
\end{gathered}
$$

with the initial conditions

$$
\begin{gathered}
\frac{T_{i}-\hat{T}_{i-1}}{\hat{T}_{i}-\hat{T}_{i-1}}=\eta_{i}, \\
\hat{Z}_{1}^{0}=\hat{Z}_{1}(0, \xi)=\frac{H k_{1}\left(\hat{T}_{1}-\hat{T}_{0}\right)}{k_{1}\left(\hat{T}_{1}-\hat{T}_{0}\right)+k_{2}\left(\hat{T}_{2}-\hat{T}_{1}\right)}
\end{gathered}
$$

and the additional boundary conditions

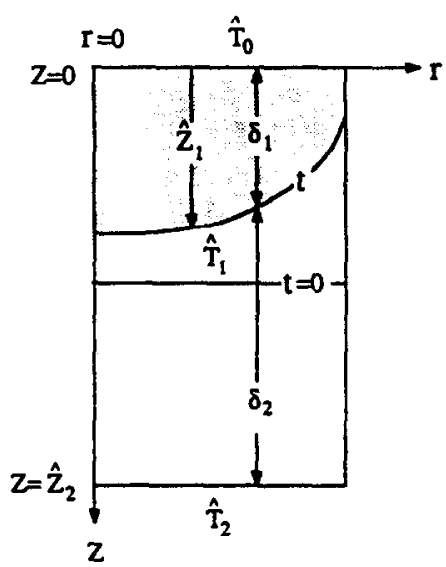

Fig. 9. Schematic diagram describing the phase-change problem in a cylindrical coordinate.

$$
\begin{aligned}
& T_{1}(t, \xi, 0)=\hat{T}_{0}, \quad T_{2}(t, \xi, 1)=\dot{T}_{2} \\
& \frac{\partial T_{i}}{\partial \zeta}\left(t, 0, \eta_{i}\right)=0, \quad \frac{\partial \hat{Z}_{1}}{\hat{C} \xi}(t, 0)=0 \\
& -\xi \delta_{i} k_{i} \frac{\hat{c} T_{i}}{\hat{c} \xi}+\xi \beta_{i} k_{i} \frac{\hat{c} T_{i}}{\hat{c} \eta_{i}}=\xi \delta_{i} h_{x_{x}}\left(T_{i}-T_{x_{x}}\right) \text { at } \xi=l \\
& k_{2}\left(\frac{\partial T_{2}}{\partial \eta_{2}}\right) \frac{\partial \hat{Z}_{1}}{\hat{c} \xi}=\delta h_{x}\left(\hat{T}_{1}-T_{x}\right) \\
& \text { at } \xi=l \text { and } \eta_{2}=0
\end{aligned}
$$

where $i=1,2$. A sample solution is obtained for the following case (the same as case $A$ in ref. [15] and this special case is chosen because an analytical solution for the steady-state position of the phase boundary is possible)

$$
\begin{gathered}
\frac{k_{2}}{k_{1}}=1, \frac{x_{2}}{x_{1}}=1, \frac{h_{\times} l}{k_{1}}=3, \frac{H}{l}=1 \\
\frac{c_{1}\left(\hat{T}_{2}-\hat{T}_{0}\right)}{h_{\mathrm{sf}}}=\frac{1}{1.5}, \frac{\hat{T}_{1}-\hat{T}_{0}}{\hat{T}_{2}-\hat{T}_{0}}=0.5, \frac{T_{x}-\hat{T}_{0}}{\hat{T}_{2}-\hat{T}_{0}}=0.75 .
\end{gathered}
$$

Thirty grid points were used in the radial direction and 15 in each phase in the axial direction. Table 5 shows a comparison of the steady-state phase boundary position calculated by the present numerical method with that obtained both analytically and numerically in ref. [15]. The present numerical method gives exceptionally accurate results as evidenced in Table 5. 
Table 5. Comparison of numerical solutions for steady-state location of freezing front with analytic solution [15]

\begin{tabular}{ccccccc}
\hline$r$ & 0.0 & 0.2 & 0.4 & 0.6 & 0.8 & 1.0 \\
\hline Analytic [15] & 0.4687 & 0.4654 & 0.4546 & 0.4317 & 0.3845 & 0.2737 \\
This work & 0.4686 & 0.4654 & 0.4545 & 0.4316 & 0.3845 & 0.2737 \\
Numerical [15] & 0.4710 & 0.4676 & 0.4564 & 0.4328 & 0.3845 & 0.2711 \\
\hline
\end{tabular}

In Fig. 10, the dependence of the transient position of the phase boundary on the dimensionless time $\tau$ $\left(=x_{1} t / l^{2}\right)$ is shown. The results of this study compare favorably with those of Duda et al. [15]. since the largest difference that occurs at the steady-state limit (the numerical values of the position of the phase boundary corresponding to this time are tabulated in Table 5) is within $1 \%$.

\section{SUMMARY}

A finite-difference method based on a coordinate transformation is developed for phase-change problems with multiple moving boundaries of irregular shape. The coordinate transformation employed here preserves and utilizes the conservative forms so the conservation principle is obeyed exactly in each phase.
The resulting discretization equations are associated with the moving control volume which undergoes stretching/contraction (also distortion in two-dimensional problems) in the physical coordinates. The moving boundaries are treated explicitly to avoid iterations, while the temperature field equations are treated implicitly. The inconsistent heat fluxes, occurring at the interfaces due to the explicit treatment of the interfacial energy balances, are used to update the positions of boundaries, while the mass fluxes are always conserved throughout all phases.

The accuracy, which is an important criterion for numerical methods, is investigated by solving some specific problems with large density change, heat generation, and multiple moving boundaries. The present numerical method gives rise to good or better results. when compared with available semi-analytical and

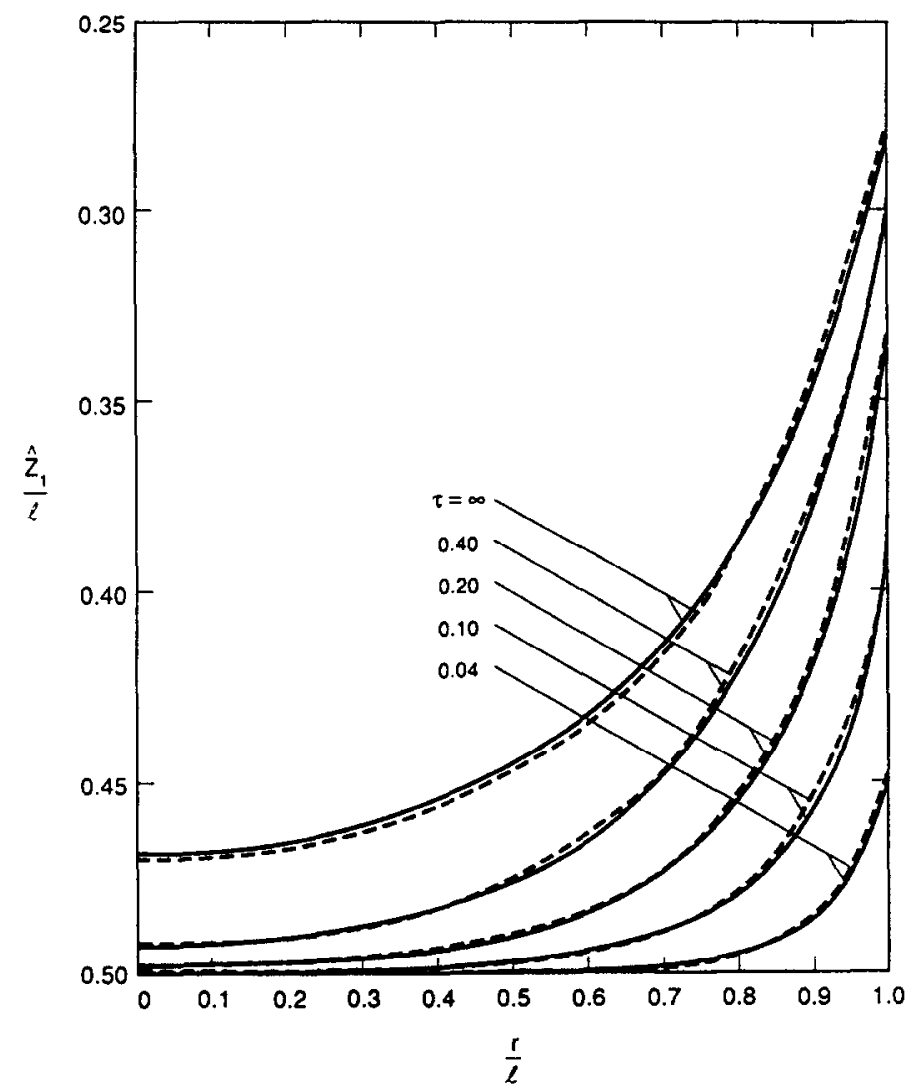

FIG. 10. Comparison of numerical solution for transient position of the phase boundary. This work. Duda et al. [15], 
numerical solutions. The present numerical method proves to be fairly general and flexible and can be used for a wide range of phase-change problems where phase change occurs at a distinct temperature. For the problems where the latent heat is released over a range of temperatures, the present numerical method is not applicable. Although the solution method is tested against phase-change problems, it is equally applicable to moving boundary problems without phase changes.

Acknowledgements-The financial support of NASA (Grant: NAG3-850 through Lewis Research Center) is gratefully acknowledged. Mr Terry Hardy (project monitor) and Mr Richard DeWitt (program manager) of the Slush Hydrogen Technology Project of NASP have made valuable recommendations for which we are also grateful.

\section{REFERENCES}

1. H. S. Carslaw and J. C. Jaeger, Conduction of Heat in Solids. Clarendon Press, Oxford (1959).

2. T. R. Goodman, Heat balance integral-further considerations and refinements, I. Heat Transfer 83, 83-86 (1961).

3. M. A. Biot and H. Daughaday, Variation analysis of ablation, J. Aerospace Sci. 29, 227-229 (1962).

4. B. A. Boley and H. P. Yagoda. The starting solutions for two-dimensional heat conduction problems with phase change, $Q$. Appl. Math. 27, 223-246 (1969).

5. R. I. Pedroso and G. A. Domoto, Perturbation solutions for spherical solidification of saturated liquids, J. Heat Transfer 95, 42-46 (1973).

6. R. I. Pedroso and G. A. Domoto. Exact solution by perturbation method for planar solidification of a saturated liquid with convection at the wall, $I n t$. J. Heat . Lass Transfer 16, 1816-1819 (1973).

7. N. Shamsundar and E. M. Sparrow, Effect of density change on multidimensional conduction phase change, J. Heat Transfer 97, 550-557 (1976).

8. A. B. Crowley, Numerical solution of Stefan problems. Int. J. Heat Mass Transfer 21, 215-219 (1978).

9. C. Bonacina, G. Comini, A. Fasano and M. Primicerio, Numerical solution of phase-change problems, $I n t . J$. Heat Mass Transfer 16, 1825-1832 (1973).

10. J. Crank and R. S. Gupta, Isotherm migration method in two dimensions. Int. J. Heat Mass Transfer 18. $1101-$ 1117 (1975).

11. E. M. Sparrow and W. Chuck. An implicit explicit numerical solution scheme for phase-change problems, Numer. Heat Transfer 7, 1-15 (1984).

12. E. M. Sparrow, S. Ramadhyani and S. V. Patankar, Effect of subcooling on cylindrical melting, $J$. Heat Transfer 100, 395-402 (1978).

13. F. B. Cheung. T. C. Chawla and D. R. Pedersen, The effects of heat generation and wall interaction on freezing and melting in a finite slab, Int. J. Heat Mass Transfer 27, 29-37 (1984).

14. T. Saitoh, Numerical method for multi-dimensional freezing problems in arbitrary domains, $J$. Heat Transfer 100, 294-299 (1978).

15. J. L. Duda, M. F. Malone. R. H. Notter and J. S. Vrentas, Analysis of two-dimensional diffusioncontrolled moving boundary problems, Int. J. Heat Mass Transfer 18, 901-910 (1975).

16. C. F. Hsu, E. M. Sparrow and S. V. Patankar, Numerical solution of moving boundary problems by boundary immobilization and a control-volume-based finitedifference scheme. Int. J. Heat Mass Transfer 24, $1335-$ $1343(1981)$

17. J. Crank, Free and Mocing Boundary Problems. Clarendon Press. Oxford (1984).

18. S. V. Patankar, Numerical Heat Transfer and Fluid Flou. Hemisphere, Washington, DC (1980).

19. V. S. Arpaci and P. S. Larsen. Concection Heat Transfer. Prentice-Hall. Englewood Cliffs. New Jersey (1984).

20. C.-J. Kim. M. Kaviany and R. Sonntag. Analysis of freezing of bubbles in cryogenic gel production, 26th ASME/AIChE Natn. Heat Transfer Conf., Philadelphia, 69 August. 89-HT-9 (1989).

21. L. E. Scriven, On the dynamics of phase growth, Chem. Engng Sci. 10, 1-13 (1959).

22. J. M. Hill and A. Kucera. Freezing a saturated liquid inside a sphere. Int. J. Heat Mass Transter 26, 16311637 (1983).

23. L. C. Tao, Generalized numerical solutions of freezing a saturated liquid in cylinders and spheres. A.I.Ch.E. $J$ 13, 165-169 (1967).

24. K. C. Karki and S. V. Patankar, Calculation procedure for viscous incompressible flows in complex geometries, Numer. Heat Transfer 14, 295-307 (1988)

25. D. N. de G. Allen and R. T. Severn. The application of relaxation methods to the solution of non-elliptic partial differential equations, Q.J. Mech. Appl. Math. 15, 5362 (1962).

26. A. Lazaridis. A numerical solution of the multi-dimensional solidification (or melting) problems. $\mathrm{lnt}$. J. Heat Mass Transfer 13, 1459-1477 (1970).

\title{
UNE METHODE NUMERIQUE POUR LES PROBLEMES DE CHANGEMENT DE PHASE
}

\begin{abstract}
Résumé-On développe une mèthode précise aux différences finies pour les problèmes de changement de phase avec plusieurs frontières mobiles de forme irrégulière, en employant une transformation de coordonnèes qui immobilise les frontières mobiles et préserve les formes conservatives des équations originales. La méthode est tout d'abord présentée sur des problèmes monodimensionnels (avec grande variation de densité entre les phases, génération de chaleur, et plusieurs frontières mobiles) puis elle est étendue aux problèmes bidimensionnels (sans changement de densité entre phase). Des solutions numériques sont obtenues de façon non itératives en utilisant un traitement explicite des bilans de masse et d'énergie et un traitement implicite des équations de champ de température. La précision et la flexibilité de la méthode numérique présentée sont vérifiées en résolvant quelques problèmes de changement de phase et en comparant les résultats avec les solutions analytiques, semi-analytiques et numériques existantes. Les résultats montrent que des problèmes mono ou bidimensionnels de changement de phase peuvent être traités aisément avec une excellente précision.
\end{abstract}




\title{
EIN NUMFRISCHES VERFAHREN FÜR PHASENÄNDERUNGSPROBLEME
}

Zusammenfassung-Für Phasenänderungsprobleme mit mehreren beweglichen, unregelmäßig geformten Grenzen wird ein sehr genaues und effizientes Finite-Differenzen-Verfahren entwickelt. Eine KoordinatenTransformation hält die beweglichen Grenzen fest und bewahrt die Form der ursprünglich gültigen Gleichungen. Das numerische Verfahren wird zunächst für eindimensionale Phasenänderungsprobleme dargestellt (einschließlich großer Dichteunterschiede zwischen den Phasen, Wärmeerzeugung und mehreren beweglichen Grenzen). Anschließend wird es auf zweidimensionale Probleme erweitert (ohne Dichteänderung zwischen den Phasen). Die numerischen Lösungen ergeben sich aus einer expliziten Behandlung der Stoff- und Energiebilanz an der Grenzfläche und einer impliziten Behandlung der Feldgleichungen für die Temperatur. Dic Genauigkeit und Anpassungsfähigkeit der vorliegenden numerischen Verfahren werden durch die Lösung einiger Phasenänderungsprobleme und durch den Vergleich mit vorhandenen analytischen, halb-analytischen und numerischen Lösungen verifiziert. Die Ergebnisse zeigen, da $B$ ein- und zweidimensionale Phasenänderungsprobleme mit sehr guter Genauigkeit berechnet werden können.

\section{ЧИСЛЕННЫЙ МЕТОДА РЕШЕНИЯ ЗАДАЧ ФАЗОВОГО ПЕРЕХОДА}

\begin{abstract}
Aнаотаит-Разработан достаточно точный и эффехтивный конечно-разностный метод решения задач фазового перехода с многочнслеными двихушимися границами неправильной формы на основе гоординатного преобразования, фихсирующего двихушиеся границы и сохраняющего хонсеративную форму исходных определяоших уравненнй. Чнсленньй метод сначала представлен для одномерных задач фазового перехода (учитывающих существенное изменение плотностей мехду фазами, тепловыделение и неединственность двихуцейся границы), а затем расширен пля решения двумерных задач (без изменения плотностей мехду фазами). Чнсленные результаты получены неитеративным методом преобразования мехфазных балаисов массы и энергии на основе явной схемы и преобразования уравнений для температурных полей с использованием неявной схемы. Точность и гибкость предлохенного численного метода проверяется решением некоторых задач с фазовыми переходами и сравненмем полученных результатов с имеюшимнся аналитическими, полуаналитическими и численными решениями. Результаты свидетельствуют о том, что одно- и двумерные задачи фазового перехода могут решаться легхо и с высохой точностью.
\end{abstract}

